\title{
AN EIGENVALUE PINCHING THEOREM FOR COMPACT HYPERSURFACES IN A SPHERE
}

\author{
YINGXIANG HU AND HONGWEI XU
}

\begin{abstract}
In this article, we prove an eigenvalue pinching theorem for the first eigenvalue of the Laplacian on compact hypersurfaces in a sphere. Let $\left(M^{n}, g\right)$ be a closed, connected and oriented Riemannian manifold isometrically immersed by $\phi$ into $\mathbb{S}^{n+1}$. Let $q>n$ and $A>0$ be some real numbers satisfying $|M|^{\frac{1}{n}}\left(1+\|B\|_{q}\right) \leq A$. Suppose that $\phi(M) \subset B\left(p_{0}, R\right)$, where $p_{0}$ is a center of gravity of $M$ and radius $R<\frac{\pi}{2}$. We prove that there exists a positive constant $\varepsilon$ depending on $q, n, R$ and $A$ such that if $n\left(1+\|H\|_{\infty}^{2}\right)-\varepsilon \leq \lambda_{1}$, then $M$ is diffeomorphic to $\mathbb{S}^{n}$. Furthermore, $\phi(M)$ is starshaped with respect to $p_{0}$, Hausdorff close and almost-isometric to the geodesic sphere $S\left(p_{0}, R_{0}\right)$, where $R_{0}=\arcsin \frac{1}{\sqrt{1+\|H\|_{\infty}^{2}}}$.
\end{abstract}

\section{INTRODUCTION}

The eigenvalue problem for compact hypersurfaces in a sphere is a subject of particular interest in geometry of submanifolds. Throughout this article, let $\left(M^{n}, g\right)$ be a closed, connected and oriented $n(\geq 2)$-dimensional Riemannian manifold isometrically immersed by $\phi$ into the unit sphere $\left(\mathbb{S}^{n+1}\right.$, can $)$, i.e., $\phi^{*} c a n=g$. When $M^{n}$ is a minimal hypersurface in $\mathbb{S}^{n+1}$, it follows from Takahashi's theorem [22] that the first nonzero eigenvalue $\lambda_{1}(M)$ of the Laplacian of $\left(M^{n}, g\right)$ is not greater than $n$. Inspired by this, Yau [25] conjectured that for every closed embedded minimal hypersurface $M^{n}$ of $\mathbb{S}^{n+1}, \lambda_{1}(M)=n$. Up to now, Yau's conjecture is far from being solved. The first breakthrough to this conjecture was made by Choi and Wang [4]. They proved that $\lambda_{1}(M) \geq \frac{n}{2}$. A careful argument (see [2], Theorem 5.1) shows that the strict inequality holds, i.e., $\lambda_{1}(M)>\frac{n}{2}$. More recently, Tang and Yan [23] made a new breakthrough and proved that Yau's conjecture is true for closed minimal isoparametric hypersurfaces of $\mathbb{S}^{n+1}$. For further discussions, we refer to [24].

Besides the minimal case, by considering the canonical embedding of $\mathbb{S}^{n+1}$ into $\mathbb{R}^{n+2}$ and applying the Reilly's inequality [15] for the Euclidean submanifolds, one can easily obtain

$$
\lambda_{1}(M) \leq n\left(1+\|H\|_{2}^{2}\right) .
$$

2010 Mathematics Subject Classification. 53C20, 53C40, 58C40.

Key words and phrases. Compact hypersurfaces, first eigenvalue, differentiable pinching theorem, mean curvature, Hausdorff distance.

Research supported by the National Natural Science Foundation of China, Grant Nos. 11371315,11201416 
Here the $L^{p}$-norm is always normalized, i.e., for any $f \in C^{\infty}(M)$,

$$
\|f\|_{p}:=\left(\frac{1}{|M|} \int_{M}|f|^{p} d v\right)^{\frac{1}{p}},
$$

where $|M|, d v$ are the Riemannian volume and volume element of $\left(M^{n}, g\right)$ respectively. From the Hölder inequality, it follows that for $p \leq q$, we have $\|f\|_{p} \leq\|f\|_{q}$. Furthermore, $\lim _{p \rightarrow \infty}\|f\|_{p}=\|f\|_{\infty}$.

In [6], Grosjean showed that the equality (1.1) holds if and only if $\phi$ is minimal or $\phi(M)$ is a geodesic hypersphere of $\mathbb{S}^{n+1}$. Moreover, if $\phi(M)$ is contained in an open hemisphere, the equality holds if and only if $\phi(M)$ is a geodesic hypersphere of $\mathbb{S}^{n+1}$. This follows from the fact [13] that there exists no closed minimal hypersurface in an open hemisphere. Motivated by the rigidity result, it is natural to investigate the eigenvalue pinching problem for compact hypersurfaces by relaxing the equality (1.1).

We denote the open (resp. closed) geodesic ball of center $p$ and radius $R$ by $B(p, R)$ (resp. $\bar{B}(p, R)$ ), and the geodesic sphere of center $p$ and radius $R$ by $S(p, R)$. In 2012, Grosjean and Roth [7] studied the eigenvalue pinching problem for the compact hypersurface of ambient spaces with bounded sectional curvature. When the ambient space is restricted to $\mathbb{S}^{n+1}$, their result (see [7], Corollary 1.1) can be stated as follows.

Theorem 1.1. Let $\left(M^{n}, g\right)(n \geq 2)$ be a closed, connected and oriented Riemannian manifold isometrically immersed by $\phi$ into $\mathbb{S}^{n+1}$. Assume that $|M| \leq c \omega_{n}$ with $c \in(0,1)$ and $\phi(M)$ lies in a geodesic ball of radius $\frac{\pi}{8}$. Let $p_{0}$ be the center of mass of $M$. Let $\varepsilon<\frac{1}{6}, q>n$ and $A>0$ be some real numbers satisfying $\max \left(|M|^{\frac{1}{n}}\|H\|_{\infty},|M|^{\frac{1}{n}}\|B\|_{q}\right) \leq A$. Then there exist positive constants $C=C(q, n, A)$ and $\alpha=\alpha(q, n)$ such that if

$$
n\left(1+\|H\|_{\infty}^{2}\right) \leq \lambda_{1}(M)(1+\varepsilon),
$$

holds with $C \varepsilon^{\alpha}<1$, then

$$
d_{H}\left(\phi(M), S\left(p_{0}, R_{0}\right)\right) \leq \frac{C \varepsilon^{\alpha}}{\sqrt{1+\|H\|_{\infty}^{2}}},
$$

where $d_{H}$ denotes the Hausdorff distance and $R_{0}=\arcsin \frac{1}{\sqrt{1+\|H\|_{\infty}^{2}}}$. Furthermore, $M$ is diffeomorphic to $\mathbb{S}^{n}$ and almost-isometric to $S\left(p_{0}, R_{0}\right)$.

A natural question is: to what extent can one enlarge the geodesic ball in Theorem 1.1? In this article, we will prove the following eigenvalue pinching theorem for compact hypersurfaces in a sphere.

Theorem 1.2. Let $\left(M^{n}, g\right)(n \geq 2)$ be a closed, connected and oriented Riemannian manifold isometrically immersed by $\phi$ into $\mathbb{S}^{n+1}$. Let $p_{0}$ be a center of gravity of $M$. Let $q>n$ and $A>0$ be some real numbers satisfying $|M|^{\frac{1}{n}}\left(1+\|B\|_{q}\right) \leq A$. Suppose that $\phi(M) \subset \bar{B}\left(p_{0}, R\right)$ with $R<\frac{\pi}{2}$ and set $\beta=\cot (R)$. There exists an explicit positive constant $\varepsilon_{0}=\varepsilon_{0}(q, n, \beta, A)$ such that if

$$
n\left(1+\|H\|_{\infty}^{2}\right)-\varepsilon \leq \lambda_{1}(M)
$$

holds with $\varepsilon<\varepsilon_{0}$, then $M$ is diffeomorphic to $\mathbb{S}^{n}$. Moreover,

(i) $\phi(M)$ is starshaped with respect to $p_{0}$; 
(ii) There exists a positive constant $D=D(n, \beta, A)$ such that

$$
d_{H}\left(\phi(M), S\left(p_{0}, R_{0}\right)\right) \leq \frac{D \varepsilon^{\frac{1}{2(2 n+1)}}}{\sqrt{1+\|H\|_{\infty}^{2}}} ;
$$

(iii) $M$ is almost-isometric to $S\left(p_{0}, R_{0}\right)$. More precisely, there exist positive constants $D_{1}=D_{1}(n, \beta, A)$ and $D_{2}=D_{2}(q, n, \beta, A)$ and a diffeomorphism $F$ : $\left(M, d_{1}\right) \rightarrow\left(S\left(p_{0}, R_{0}\right), d_{2}\right)$ such that

$$
\left|d_{2}\left(F\left(x_{1}\right), F\left(x_{2}\right)\right)-d_{1}\left(x_{1}, x_{2}\right)\right| \leq \frac{\max \left\{D_{1} \varepsilon^{\frac{1}{2(2 n+1)}}, D_{2} \varepsilon^{\frac{q-n}{q-n+q n}}\right\}}{\sqrt{1+\|H\|_{\infty}^{2}}},
$$

for any $x_{1}, x_{2} \in M$, and $d_{1}, d_{2}$ are the standard distance functions on $M$ and $S\left(p_{0}, R_{0}\right)$ respectively.

Remark 1.1. It should be mentioned that our result is proved without the assumption on volume of hypersurfaces. In Section 5, we construct an example to show that the condition on radius of geodesic ball is optimal. For other pinching results on compact hypersurfaces, we refer readers to $[1,5,10,16,17,18]$.

\section{Notations And Lemmas}

First of all, we recall that the Hausdorff distance between two compact subsets $A$ and $B$ of a metric space $(M, d)$ is given by

$$
d_{H}(A, B)=\inf \left\{\eta>0 \mid A \subset V_{\eta}(B) \text { and } B \subset V_{\eta}(A)\right\},
$$

where for any subset $A \subset M, V_{\eta}(A)$ is the tubular neighborhood of $A$ defined by $V_{\eta}(A):=\{x \in M \mid d(x, A)<\eta\}$.

Let $\left(X, d_{X}\right)$ and $\left(Y, d_{Y}\right)$ be two metric spaces. Given a real number $\theta>0$, a $\theta$-isometry (almost isometry) is a map $F:\left(X, d_{X}\right) \rightarrow\left(Y, d_{Y}\right)$ satisfying

(1) for any $x_{1}, x_{2} \in X,\left|d_{Y}\left(F\left(x_{1}\right), F\left(x_{2}\right)\right)-d_{X}\left(x_{1}, x_{2}\right)\right|<\theta$;

(2) for any point $y \in Y$, there exists a point $x \in X$ with $d_{Y}(y, F(x))<\theta$.

Let $\left(M^{n}, g\right)$ be a closed, connected and oriented $n$-dimensional Riemannian manifold isometrically immersed by $\phi$ in $\left(\mathbb{S}^{n+1}\right.$, can $)$. In the sequel, we denote by $\nabla$ and $\bar{\nabla}$ the gradient associated to $g$ and can respectively. For any $p_{0} \in \mathbb{S}^{n+1}$, let $\exp _{p_{0}}$ be the exponential map at this point and $r(x):=d\left(p_{0}, x\right)$ be the distance function to this point. We denote by $\left(x_{i}\right)_{1 \leq i \leq n+1}$ the normal coordinates of $\mathbb{S}^{n+1}$ centered at $p_{0}$. The position vector $X$ is defined by $X:=\sin r \bar{\nabla} r$, and it is easy to observe that the normal coordinates of $X$ are $\frac{\sin r}{r} x_{i}$. We will use $\frac{\sin r}{r} x_{i}$ as test functions in the variational characterization of $\lambda_{1}(M)$ but the mean of these functions must be zero. For this purpose, there are two ways to choose a specific point $p_{0} \in \mathbb{S}^{n+1}$. One is the center of mass of $M$, which is given by Chavel [3] and Heintze [8]. Indeed, we assume that $\phi(M)$ is contained in an open hemisphere of $\mathbb{S}^{n+1}$. Let $Y$ be the vector field in this open hemisphere defined by

$$
Y_{q}=\int_{M} \frac{\sin d(q, x)}{d(q, x)} \exp _{q}^{-1}(x) d v(x) \in T_{q} \mathbb{S}^{n+1},
$$

then by Brouwer's fixed point theorem, there exists a point $p_{0}$ in this open hemisphere such that $Y_{p_{0}}=0$. Hence for this $p_{0}$, we have $\int_{M} \frac{\sin r}{r} x_{i} d v=0$. The other 
one is the center of gravity of $M$, which is introduced by Veeravalli [21]. It is defined as a critical point of the smooth function

$$
\mathcal{E}: \mathbb{S}^{n+1} \rightarrow \mathbb{R}, \quad p \mapsto \int_{M}[1-\cos d(p, x)] d v(x) .
$$

The introduction of $\cos (\cdot)$ has a significant advantage: the distance function $d(p, \cdot)$ may be non-smooth at some points, but thanks to $\cos (\cdot), \mathcal{E}$ is smooth on the whole manifold $\mathbb{S}^{n+1}$. Furthermore, by the compactness of $\mathbb{S}^{n+1}$, there exist at least two centers of gravity. If $p_{0} \in \mathbb{S}^{n+1}$ is a critical point of $\mathcal{E}$, then for any unit vector $u \in T_{p_{0}} \mathbb{S}^{n+1}$ we have

$$
0=\left\langle\bar{\nabla} \mathcal{E}\left(p_{0}\right), u\right\rangle=-\int_{M}\langle\bar{\nabla} \cos r(x), u\rangle d v(x),
$$

which indicates that $\int_{M} \frac{\sin r}{r} x_{i} d v=0$. Therefore, a center of gravity of $M$ is also a center of mass of $M$. In the sequel, we assume that $\phi(M)$ contained in $\bar{B}\left(p_{0}, R\right)$, where $p_{0}$ is a center of gravity of $M$ and radius $R<\frac{\pi}{2}$. We recall two lemmas shown by Heintze [8].

Lemma 2.1. At any $x \in M$, we have

$$
\sum_{i=1}^{n+1}\left|\nabla\left(\frac{\sin r}{r} x_{i}\right)\right|^{2}=n-\left|X^{\top}\right|^{2} .
$$

Lemma 2.2. The vector field $X=\sin r \bar{\nabla} r$ satisfies

$$
\operatorname{div}\left(X^{\top}\right)=n \cos r-n H\langle X, \nu\rangle .
$$

We also recall an inequality given by Jorge and Xavier [11], which relates the extrinsic radius and the mean curvature. If $\phi(M)$ lies in a closed geodesic ball of radius $R<\frac{\pi}{2}$, then $\|H\|_{\infty} \geq \cot (R)=$ : $\beta$. By using the identity (2.2) and $X^{\top}=\sin r \nabla r$, we obtain

$$
\begin{aligned}
\frac{1}{|M|} \int_{M}\left(n-\left|X^{\top}\right|^{2}\right) d v & =\frac{1}{|M|} \int_{M}\left(n \sin ^{2} r+n H\langle X, \nu\rangle \cos r\right) d v \\
& \leq \frac{n}{|M|} \int_{M} \sin ^{2} r d v+\frac{n\|H\|_{\infty}}{|M|} \int_{M} \sin r \cos r d v \\
& =n\|X\|_{2}^{2}+\frac{\|H\|_{\infty}}{|M|} \int_{M} \sin r\left(\operatorname{div}\left(X^{\top}\right)+n H\langle X, \nu\rangle\right) d v \\
& \leq n\left(1+\|H\|_{\infty}^{2}\right)\|X\|_{2}^{2}-\frac{\|H\|_{\infty}}{|M|} \int_{M} \cot r\left|X^{\top}\right|^{2} d v
\end{aligned}
$$

Since $\cot (\cdot)$ is decreasing on $\left[0, \frac{\pi}{2}\right)$, we get

$$
1 \leq\left(1+\|H\|_{\infty}^{2}\right)\|X\|_{2}^{2}+\frac{1}{n}\left(1-\beta^{2}\right)\left\|X^{\top}\right\|_{2}^{2} .
$$

Since $p_{0}$ is a center of gravity of $M$, we may take $\left\{\frac{\sin r}{r} x_{i}\right\}_{1 \leq i \leq n+1}$ as test functions. By the identity (2.1) and the variational characterization of the first nonzero eigenvalue, we get

$$
\lambda_{1}(M)\|X\|_{2}^{2} \leq \frac{1}{|M|} \int_{M}\left(n-\left|X^{\top}\right|^{2}\right) d v \leq n\left(1+\|H\|_{\infty}^{2}\right)\|X\|_{2}^{2}-\beta^{2}\left\|X^{\top}\right\|_{2}^{2} .
$$




\section{AN $L^{2}$-ApProACH}

We consider the composition of isometric immersions $M^{n} \stackrel{\phi}{\hookrightarrow} \mathbb{S}^{n+1} \stackrel{i}{\hookrightarrow} \mathbb{R}^{n+2}$, where $i$ is the the canonical embedding of $\mathbb{S}^{n+1}$ into $\mathbb{R}^{n+2}$. We denote by $\bar{H}$ the mean curvature of $i \circ \phi$, then $|\bar{H}|^{2}=1+|H|^{2}$. Applying the Hoffman-Spruck Sobolev inequality [9] to the isometric immersion $i \circ \phi: M^{n} \rightarrow \mathbb{R}^{n+2}$, we immediately derive a Sobolev-type inequality for closed hypersurfaces in $\mathbb{S}^{n+1}$.

Lemma 3.1. Let $\left(M^{n}, g\right)(n \geq 2)$ be a closed hypersurface isometrically immersed in $\mathbb{S}^{n+1}$. For a nonnegative function $f \in C^{1}(M)$, we have

$$
\left(\int_{M} f^{\frac{n}{n-1}} d v\right)^{\frac{n-1}{n}} \leq K(n) \int_{M}\left(|\nabla f|+f \sqrt{1+|H|^{2}}\right) d v,
$$

where $K(n)$ is a positive constant depending only on $n$.

Now we give some $L^{2}$-estimates under the eigenvalue pinching condition. For simplicity, we denote by $\left(\Lambda_{\varepsilon}\right)$ the pinching condition $n\left(1+\|H\|_{\infty}^{2}\right)-\varepsilon \leq \lambda_{1}(M)$. We also abbreviate $\lambda_{1}=\lambda_{1}(M), h=\sqrt{1+\|H\|_{\infty}^{2}}$. Moreover, we omit the volume element $d v$ in the integrals when there is no confusion. First, we have

Lemma 3.2. If $\left(\Lambda_{\varepsilon}\right)$ holds, then

$$
\left\|X^{\top}\right\|_{2}^{2} \leq \frac{\varepsilon}{\beta^{2}}\|X\|_{2}^{2}
$$

Proof. By $(2.4)$ and $\left(\Lambda_{\varepsilon}\right)$, we have

$$
n h^{2}-\varepsilon \leq n h^{2}-\beta^{2} \frac{\left\|X^{\top}\right\|_{2}^{2}}{\|X\|_{2}^{2}}
$$

which gives the desired estimate.

Since $\left\|X^{\top}\right\|_{2}^{2} \leq\|X\|_{2}^{2}$, it is natural to assume that $\varepsilon \leq \beta^{2}$.

Lemma 3.3. If $\left(\Lambda_{\varepsilon}\right)$ holds with $\varepsilon \leq \beta^{2}$, then

$$
\frac{n}{(n+1) h^{2}} \leq\|X\|_{2}^{2} \leq \frac{1+\varepsilon}{h^{2}} \leq 1 .
$$

Proof. Since $\varepsilon \leq \beta^{2} \leq \frac{n}{2} \beta^{2} \leq \frac{n h^{2}}{2}$, then $\left(\Lambda_{\varepsilon}\right)$ implies that $\lambda_{1} \geq \frac{n h^{2}}{2} \geq \frac{n}{2} \geq 1$. Then by (2.4) we get

$$
\begin{aligned}
n h^{2}\|X\|_{2}^{2} & \leq\left(\lambda_{1}+\varepsilon\right)\|X\|_{2}^{2} \leq \lambda_{1}(1+\varepsilon)\|X\|_{2}^{2} \\
& \leq(1+\varepsilon)\left(n-\left\|X^{\top}\right\|_{2}^{2}\right) \leq n(1+\varepsilon),
\end{aligned}
$$

which proves the right-hand side of (3.3). On the other hand, by (2.3) and Lemma 3.2 , we have

$$
1 \leq h^{2}\|X\|_{2}^{2}+\frac{1}{n}\left\|X^{\top}\right\|_{2}^{2} \leq h^{2}\left(1+\frac{\varepsilon}{n \beta^{2}}\right)\|X\|_{2}^{2} \leq \frac{(n+1) h^{2}}{n}\|X\|_{2}^{2} .
$$

Let $Y=n \cos r H \nu-n\|H\|_{\infty}^{2} X$.

Lemma 3.4. If $\left(\Lambda_{\varepsilon}\right)$ holds with $\varepsilon \leq \beta^{2}$, then

$$
\|Y\|_{2}^{2} \leq 4 n^{2} h^{2} \varepsilon\left(1+\frac{1}{\beta^{2}}\right) .
$$


Proof. By the identity (2.2), Lemmas 3.2 and 3.3, we have

$$
\begin{aligned}
\|Y\|_{2}^{2} & =\frac{n^{2}}{|M|} \int_{M} H^{2} \cos ^{2} r-\frac{2 n^{2}\|H\|_{\infty}^{2}}{|M|} \int_{M} H\langle X, \nu\rangle \cos r+n^{2}\|H\|_{\infty}^{4}\|X\|_{2}^{2} \\
& \leq-n^{2}\|H\|_{\infty}^{2}\left(1-\|X\|_{2}^{2}\right)+2 n\|H\|_{\infty}^{2}\left\|X^{\top}\right\|_{2}^{2}+n^{2}\|H\|_{\infty}^{4}\|X\|_{2}^{2} \\
& \leq n^{2}\|H\|_{\infty}^{2} \varepsilon+4 n^{2}\|H\|_{\infty}^{2} \frac{\varepsilon}{\beta^{2}} \\
& \leq 4 n^{2} h^{2} \varepsilon\left(1+\frac{1}{\beta^{2}}\right) .
\end{aligned}
$$

Let $W=|X|^{-\frac{1}{2}}(X|X|+|X| \cos r H \nu-h X)$.

Lemma 3.5. If $\left(\Lambda_{\varepsilon}\right)$ holds with $\varepsilon \leq \beta^{2}$, then

$$
\|W\|_{2}^{2} \leq 4 h \varepsilon\left(1+\frac{1}{\beta^{2}}\right) .
$$

Proof. By Lemmas 3.2 and 3.3, a similar computation gives

$$
\begin{aligned}
\|W\|_{2}^{2} & \leq \frac{1}{|M|} \int_{M}\left[|X \| X+H \cos r \nu|^{2}-2 h\langle X+H \cos r \nu, X\rangle+h^{2}|X|\right] \\
& \leq \frac{1}{|M|} \int_{M}\left[|X \| X+H \cos r \nu|^{2}-2 h\langle X+H \cos r \nu, X\rangle\right]+h^{2}\|X\|_{2} \\
& \leq 2 h^{2}\|X\|_{2}-2 h+\frac{2 h}{n}\left\|X^{\top}\right\|_{2}^{2} \\
& \leq 4 h \varepsilon\left(1+\frac{1}{\beta^{2}}\right) .
\end{aligned}
$$

Lemma 3.6. Let $A>0$ be a real number such that $|M|^{\frac{1}{n}}\left(1+\|H\|_{2}\right) \leq A$. If $\left(\Lambda_{\varepsilon}\right)$ holds with $\varepsilon \leq \min \left(1, \beta^{2}\right)$, then there exists a positive constant $C_{1}=C_{1}(n, A)$ such that

$$
\|X\|_{\infty} \leq C_{1}\|X\|_{2} .
$$

Proof. Since $\varphi:=|X|=\sin r$, we have $\left|d \varphi^{2 \alpha}\right| \leq 2 \alpha \varphi^{2 \alpha-1} \cos r \leq 2 \alpha \varphi^{2 \alpha-1}$. Hence using Lemma 3.1, we get for any $\alpha \geq 1$ and $f=\varphi^{2 \alpha}$,

$$
\|\varphi\|_{\frac{2 \alpha n}{n-1}}^{2 \alpha} \leq K(n)|M|^{\frac{1}{n}}\left(2 \alpha+h\|\varphi\|_{\infty}\right)\|\varphi\|_{2 \alpha-1}^{2 \alpha-1}
$$

By Lemma 3.3, we have

$$
\|\varphi\|_{\infty} \geq\|\varphi\|_{2} \geq \frac{1}{h} \sqrt{\frac{n}{n+1}}
$$


Then taking $\nu=\frac{n}{n-1}, \alpha=\frac{a_{p}+1}{2}$, where $a_{p+1}=\left(a_{p}+1\right) \nu$ and $a_{0}=2$, we get

$$
\begin{aligned}
\left(\frac{\|\varphi\|_{a_{p+1}}}{\|\varphi\|_{\infty}}\right)^{\frac{a_{p+1}}{\nu^{p+1}}} & \leq\left[K(n)|M|^{\frac{1}{n}}\left(\frac{a_{p}+1}{\|\varphi\|_{\infty}}+h\right)\right]^{\frac{1}{\nu^{p}}}\left(\frac{\|\varphi\|_{a_{p}}}{\|\varphi\|_{\infty}}\right)^{\frac{a_{p}}{\nu^{p}}} \\
& \leq\left[5 K(n)|M|^{\frac{1}{n}} a_{p} h\right]^{\frac{1}{\nu^{p}}}\left(\frac{\|\varphi\|_{a_{p}}}{\|\varphi\|_{\infty}}\right)^{\frac{a_{p}}{\nu^{p}}} \\
& \leq\left(\prod_{i=1}^{p} a_{i}^{\frac{1}{\nu^{i}}}\right)\left[5 K(n)|M|^{\frac{1}{n}} h\right]^{n\left(1-\frac{1}{\nu^{p+1}}\right)}\left(\frac{\|\varphi\|_{a_{0}}}{\|\varphi\|_{\infty}}\right)^{a_{0}} .
\end{aligned}
$$

By $\left(\Lambda_{\varepsilon}\right)$ with $\varepsilon \leq 1$ and $(1.1)$, we see

$$
|M|^{\frac{1}{n}} h \leq|M|^{\frac{1}{n}} \sqrt{(1+\varepsilon)\left(1+\|H\|_{2}^{2}\right)} \leq \sqrt{2}|M|^{\frac{1}{n}}\left(1+\|H\|_{2}\right) \leq \sqrt{2} A .
$$

Observing that $\frac{a_{p}}{\nu^{p}}$ converges to $a_{0}+n$ and $a_{0}=2$, we get

$$
\|\varphi\|_{\infty} \leq C(n) A^{\frac{n}{2}}\|\varphi\|_{2}=: C_{1}(n, A)\|\varphi\|_{2} \text {. }
$$

Let us introduce the function $\psi:=|X|^{\frac{1}{2}}|| X\left|-\frac{1}{h}\right|=|X|^{\frac{1}{2}}\left|X-\frac{1}{h} \frac{X}{|X|}\right|$. First, we give an $L^{1}$-estimate of $\psi$.

Lemma 3.7. If $\left(\Lambda_{\varepsilon}\right)$ holds with $\varepsilon \leq \beta^{2}$, then

$$
\|\psi\|_{1} \leq \frac{5 \varepsilon^{\frac{1}{2}}}{h^{\frac{3}{2}}}\left(1+\frac{1}{\beta^{2}}\right)^{\frac{1}{2}} .
$$

Proof. First we have

$$
\begin{aligned}
\psi & =|X|^{\frac{1}{2}}\left|\frac{1}{h^{2}}\left(h^{2} X-X-H \cos r \nu\right)+\frac{1}{h^{2}}\left(X+H \cos r \nu-h \frac{X}{|X|}\right)\right| \\
& \leq \frac{|X|^{\frac{1}{2}}}{n h^{2}}|Y|+\frac{1}{h^{2}}|W|
\end{aligned}
$$

Then by the Hölder inequality, Lemmas 3.4 and 3.5, we get

$$
\begin{aligned}
\|\psi\|_{1} & \leq \frac{1}{h^{2}}\left(\frac{1}{n}\|X\|_{2}^{\frac{1}{2}}\|Y\|_{2}+\|W\|_{2}\right) \\
& \leq \frac{1}{h^{2}}\left\{\frac{1}{n}\left(\frac{2}{h^{2}}\right)^{\frac{1}{4}}\left[4 n^{2} h^{2} \varepsilon\left(1+\frac{1}{\beta^{2}}\right)\right]^{\frac{1}{2}}+\left[4 h \varepsilon\left(1+\frac{1}{\beta^{2}}\right)\right]^{\frac{1}{2}}\right\} \\
& \leq \frac{5 \varepsilon^{\frac{1}{2}}}{h^{\frac{3}{2}}}\left(1+\frac{1}{\beta^{2}}\right)^{\frac{1}{2}} .
\end{aligned}
$$

Now we give an $L^{\infty}$-estimate of $\psi$.

Lemma 3.8. Let $A>0$ be a real number such that $|M|^{\frac{1}{n}}\left(1+\|H\|_{2}\right) \leq A$. If $\left(\Lambda_{\varepsilon}\right)$ holds with $\varepsilon \leq \min \left\{1, \beta^{2}\right\}$, then there exists a positive constant $C_{2}=C_{2}(n, \beta, A)$ such that

$$
\|\psi\|_{\infty} \leq \frac{C_{2} \varepsilon^{\frac{1}{2(2 n+1)}}}{h^{\frac{3}{2}}} .
$$

Furthermore, $C_{2}(n, \beta, A) \rightarrow \infty$ as $\beta \rightarrow 0$. 
Proof. Let $\alpha \geq 1$, then by Lemma 3.6 we have

$$
\begin{aligned}
\left|d \psi^{2 \alpha}\right| & =\alpha \psi^{2 \alpha-2}\left|d\left(\psi^{2}\right)\right|=\alpha \psi^{2 \alpha-2}|| X\left|-\frac{1}{h}\right||3| X\left|-\frac{1}{h}\right||d| X|| \\
& \leq 3 \alpha \psi^{2 \alpha-2}\left(\|X\|_{\infty}+\frac{1}{h}\right)^{2} \cos r \\
& \leq 3 \alpha\left(\frac{\sqrt{2} C_{1}+1}{h}\right)^{2} \psi^{2 \alpha-2} .
\end{aligned}
$$

A direct calculation gives

$$
\|\psi\|_{\infty}^{2} \leq\|X\|_{\infty}\left(\|X\|_{\infty}+\frac{1}{h}\right)^{2} \leq \frac{\sqrt{2} C_{1}\left(\sqrt{2} C_{1}+1\right)^{2}}{h^{3}} .
$$

By using Lemma 3.1, we obtain for any $\alpha \geq 1$

$$
\begin{aligned}
\|\psi\|_{\frac{2 \alpha n}{n-1}}^{2 \alpha} & \leq K(n)|M|^{\frac{1}{n}}\left[3 \alpha \frac{\left(\sqrt{2} C_{1}+1\right)^{2}}{h^{2}}+\frac{\sqrt{2} C_{1}\left(\sqrt{2} C_{1}+1\right)^{2}}{h^{2}}\right]\|\psi\|_{2 \alpha-2}^{2 \alpha-2} \\
& \leq 3 \alpha K(n)|M|^{\frac{1}{n}} h \frac{\left(\sqrt{2} C_{1}+1\right)^{3}}{h^{3}}\|\psi\|_{2 \alpha-2}^{2 \alpha-2} \\
& \leq 3 \alpha K(n) \sqrt{2} A \frac{\left(\sqrt{2} C_{1}+1\right)^{3}}{h^{3}}\|\psi\|_{2 \alpha-2}^{2 \alpha-2} .
\end{aligned}
$$

Now we put $a_{p+1}=\left(a_{p}+2\right) \nu$, with $\nu=\frac{n}{n-1}, a_{0}=1$ and $\alpha=\frac{a_{p}+2}{2}$, we get

$$
\begin{aligned}
\|\psi\|_{a_{p+1}}^{\frac{a_{p+1}}{\nu p+1}} & \left.\left.\leq\left[3 \sqrt{2} K(n) a_{p} A \frac{\left(\sqrt{2} C_{1}+1\right)^{3}}{h^{3}}\right]^{\frac{1}{\nu^{p}}}\|\psi\|_{a_{p}^{\frac{a_{p}}{\nu p}}}^{\frac{a^{p}}{\nu^{p}}}\right]^{\frac{1}{\nu^{i}}}\right)\left[3 \sqrt{2} K(n) A \frac{\left(\sqrt{2} C_{1}+1\right)^{3}}{h^{3}}\|\psi\|_{a_{0}}^{a_{0}} .\right.
\end{aligned}
$$

Note that $\frac{a_{p}}{\nu^{p}}$ converges to $a_{0}+2 n$. By Lemma 3.7, we have

$$
\|\psi\|_{\infty} \leq\left[\frac{C(n, A)}{h^{3}}\right]^{\frac{n}{2 n+1}}\left[\frac{5 \varepsilon^{\frac{1}{2}}}{h^{\frac{3}{2}}}\left(1+\frac{1}{\beta^{2}}\right)^{\frac{1}{2}}\right]^{\frac{1}{2 n+1}}=: \frac{C_{2}(n, \beta, A)}{h^{\frac{3}{2}}} \varepsilon^{\frac{1}{2(2 n+1)}} .
$$

Lemma 3.9. Let $A>0$ be a real number such that $|M|^{\frac{1}{n}}\left(1+\|H\|_{2}\right) \leq A$. There exists a positive constant $\varepsilon_{0}=\varepsilon_{0}(n, \beta, A)$ such that if $\left(\Lambda_{\varepsilon}\right)$ holds with $\varepsilon \leq \varepsilon_{0}$, then there exists a positive constant $C_{3}=C_{3}(n, \beta, A)$ satisfying

$$
|| X\left|-\frac{1}{h}\right| \leq \frac{C_{3} \varepsilon^{\frac{1}{2(2 n+1)}}}{h}, \quad \text { and } \quad\left|r-R_{0}\right| \leq C_{3} \varepsilon^{\frac{1}{2(2 n+1)}} R_{0},
$$

where $R_{0}=\arcsin \frac{1}{\sqrt{1+\|H\|_{\infty}^{2}}}$. Furthermore, $C_{3}(n, \beta, A) \rightarrow \infty$ as $\beta \rightarrow 0$. 
Proof. Consider the function $f(t):=t\left(t-\frac{1}{h}\right)^{2}$, which is increasing on $\left[0, \frac{1}{3 h}\right]$ and $\left[\frac{1}{h},+\infty\right)$, and decreasing on $\left[\frac{1}{3 h}, \frac{1}{h}\right]$. We take $\varepsilon_{0}=\varepsilon_{0}(n, \beta, A)>0$ sufficiently small such that

$$
9 C_{2}^{2} \varepsilon_{0}^{\frac{1}{2 n+1}} \leq 1, \quad \text { and } \quad \varepsilon_{0} \leq \min \left(1, \beta^{2}\right) .
$$

Then by using Lemma 3.8 and $\left(\Lambda_{\varepsilon}\right)$ with $\varepsilon \leq \varepsilon_{0}$, we see

$$
\|f(|X|)\|_{\infty}=\|\psi\|_{\infty}^{2} \leq \frac{1}{9 h^{3}}<\frac{4}{27 h^{3}}=f\left(\frac{1}{3 h}\right) .
$$

By Lemma 3.3, we have $\|X\|_{2}^{2} \geq \frac{n}{(n+1) h^{2}} \geq \frac{1}{4 h^{2}}$. Hence there exists $x_{0} \in M$ satisfying $\left|X_{x_{0}}\right| \geq \frac{1}{2 h}>\frac{1}{3 h}$. By the connectedness of $M$, it follows that $|X|>\frac{1}{3 h}$ on $M$. This implies

$$
|| X\left|-\frac{1}{h}\right| \leq \frac{\sqrt{3} C_{2}(n, \beta, A)}{h} \varepsilon^{\frac{1}{2(2 n+1)}}=: \frac{C_{3}(n, \beta, A)}{h} \varepsilon^{\frac{1}{2(2 n+1)}} .
$$

Since $|X| \leq \sin R=\frac{1}{\sqrt{1+\beta^{2}}}$ and $R_{0}=\arcsin \frac{1}{h} \geq\left(1+\frac{1}{\beta^{2}}\right)^{\frac{1}{2}} \frac{1}{h}$, we get

$$
\left|r-R_{0}\right| \leq\left(1+\frac{1}{\beta^{2}}\right)^{\frac{1}{2}} \frac{C_{3}(n, \beta, A)}{h} \varepsilon^{\frac{1}{2(2 n+1)}} \leq C_{3}(n, \beta, A) \varepsilon^{\frac{1}{2(2 n+1)}} R_{0} .
$$

\section{Proof of the Main Theorem}

Let us consider the map

$$
\begin{aligned}
F: & M \rightarrow S\left(p_{0}, R_{0}\right), \\
x & \mapsto \exp _{p_{0}}\left(R_{0} \frac{Y}{|Y|}\right),
\end{aligned}
$$

where $Y:=\exp _{p_{0}}^{-1}(x)$ and $p_{0}$ is a center of gravity of $M$.

Lemma 4.1. Let $u \in U_{x} M$ and $v=u-\langle u, \nabla r\rangle \bar{\nabla} r$. We have

$$
\left|d F_{x}(u)\right|^{2}=\frac{1}{h^{2} \sin ^{2} r}|v|^{2} .
$$

Furthermore, we have

$$
\frac{1-\|\nabla r\|_{\infty}^{2}}{h^{2} \sin ^{2} r} \leq\left|d F_{x}(u)\right|^{2} \leq \frac{1}{h^{2} \sin ^{2} r} .
$$

Proof. The proof of equality (4.1) can be found in [7]. By the construction of $v$, we immediately obtain (4.2).

Lemma 4.2. Let $q>n$ and $A>0$ be some real numbers such that $|M|^{\frac{1}{n}}(1+$ $\left.\|B\|_{q}\right) \leq A$. If $\left(\Lambda_{\varepsilon}\right)$ holds with $\varepsilon \leq \min \left(1, \beta^{2}\right)$, then there exists a positive constant $C_{4}=C_{4}(q, n, \beta, A)$ such that

$$
\left\|X^{\top}\right\|_{\infty} \leq \frac{C_{4} \varepsilon^{\frac{1}{2(1+\gamma)}}}{h}
$$

where $\gamma=\frac{q n}{q-n}$. Moreover, $C_{4}(q, n, \beta, A) \rightarrow \infty$ as $q \rightarrow n$ or $\beta \rightarrow 0$. 
Proof. Put $\chi=\left|X^{\top}\right|$. For any $\alpha \geq 1$, we have

$$
\left|d \chi^{2 \alpha}\right|=2 \alpha \chi^{2 \alpha-1}\left(\cos r|\nabla r|^{2}+\sin r|d| \nabla r||\right) .
$$

Let us estimate $|d| \nabla r||$ at a point $x \in M$. For this, let $\left\{e_{i}\right\}_{1 \leq i \leq n}$ be an orthonormal basis of $T_{x} M$. We have

$$
\begin{aligned}
|d| \nabla r \|^{2} & =\frac{1}{4|\nabla r|^{2}}\left|d\langle\bar{\nabla} r, \nu\rangle^{2}\right|^{2}=\frac{\langle\bar{\nabla} r, \nu\rangle^{2}}{|\nabla r|^{2}} \sum_{i=1}^{n}\left(\bar{\nabla} d r\left(e_{i}, \nu\right)+B\left(e_{i}, \bar{\nabla} r\right)\right)^{2} \\
& \leq \frac{2}{|\nabla r|^{2}}\left[\sum_{i=1}^{n} \bar{\nabla} d r\left(e_{i}, \nu\right)^{2}+|B|^{2}|\nabla r|^{2}\right] .
\end{aligned}
$$

Now $\sum_{i=1}^{n} \bar{\nabla} d r\left(e_{i}, \nu\right)^{2} \leq|\bar{\nabla} d r|^{2} \leq \sum_{i=1}^{n+1} \bar{\nabla} d r\left(u_{i}, u_{i}\right)^{2}$, where $\left\{u_{i}\right\}_{1 \leq i \leq n+1}$ is an orthonormal basis which diagonalizes $\bar{\nabla} d r$. From the comparison theorem for the Hessian of distance function (see [14], Theorem 27), we deduce that

$$
\sum_{i=1}^{n+1} \bar{\nabla} d r\left(u_{i}, u_{i}\right)^{2} \leq\left(\frac{\cos r}{\sin r}\right)^{2} \sum_{i=1}^{n+1}\left|u_{i}-\left\langle u_{i}, \bar{\nabla} r\right\rangle \bar{\nabla} r\right|^{2}=n\left(\frac{\cos r}{\sin r}\right)^{2} .
$$

It follows that

$$
\left.|d| \nabla r\right|^{2} \leq \frac{2 n}{|\nabla r|^{2}}\left(\frac{\cos r}{\sin r}\right)^{2}+2|B|^{2}
$$

and

$$
\begin{aligned}
\left|d \chi^{2 \alpha}\right| & \leq 2 \alpha \chi^{2 \alpha-1} C(n)\left[\cos r+\frac{\cos r}{|\nabla r|}+\sin r|B|\right] \\
& \leq 4 \alpha \chi^{2 \alpha-2} C(n)\|X\|_{\infty}\left(1+|B|\|X\|_{\infty}\right) .
\end{aligned}
$$

A straightforward calculation gives

$$
|M|^{\frac{1}{n}} h \leq \sqrt{2}|M|^{\frac{1}{n}}\left(1+\|H\|_{2}\right) \leq \sqrt{2}|M|^{\frac{1}{n}}\left(1+\|B\|_{q}\right) .
$$

By using Lemma 3.1, we get for $\alpha \geq 1$

$$
\begin{aligned}
\|\chi\|_{\frac{2 \alpha n}{n-1}}^{2 \alpha} \leq & K(n)|M|^{\frac{1}{n}}\left[4 \alpha C(n)\|X\|_{\infty}\|\chi\|_{2 \alpha-2}^{2 \alpha-2}\right. \\
& \left.+4 \alpha C(n)\|X\|_{\infty}^{2}\|B\|_{q}\|\chi\|_{\frac{(2 \alpha-2) q}{q-1}}^{2 \alpha-2}+h\|\chi\|_{2 \alpha}^{2 \alpha}\right] \\
\leq & C^{\prime}(n) \alpha\|X\|_{\infty}^{2}\left[|M|^{\frac{1}{n}} h+|M|^{\frac{1}{n}}\|B\|_{q}\right]\|\chi\|_{\frac{(2 \alpha-2) q}{q-1}}^{2 \alpha-2} \\
\leq & C(n, A) \alpha\|X\|_{\infty}^{2}\|\chi\|_{\frac{(2 \alpha-2) q}{q-1}}^{2 \alpha-2} .
\end{aligned}
$$

We take $\nu:=\frac{n(q-1)}{(n-1) q}, a_{p+1}:=a_{p} \nu+\frac{2 n}{n-1}, a_{0}=2$ and $\alpha:=\frac{q-1}{2 q} a_{p}+1$. Then $a_{p+1}=\frac{2 \alpha n}{n-1}$ and

$$
\begin{aligned}
\|\chi\|_{a_{p+1}}^{\frac{a_{p+1}}{a^{p+1}}} & \leq\left[C(n, A)\|X\|_{\infty}^{2} a_{p+1}\right]^{\frac{n}{(n-1) \nu^{p+1}}}\|\chi\|_{a_{p}^{\frac{a_{p}}{\nu p}}}^{\frac{a_{p}}{\nu^{p}}}\left[C(n, A)\|X\|_{\infty}^{2}\right]^{\frac{n}{n-1} \sum_{i=1}^{p+1} \frac{1}{\nu^{i}}}\|\chi\|_{a_{p}}^{\frac{a_{p}}{\nu^{p}}} . \\
& \leq\left(\prod_{i=1}^{p+1} a_{i}^{\frac{1}{\nu^{i}}}\right)^{\frac{n}{n-1}}[C
\end{aligned}
$$


Note that $\frac{a_{p}}{\nu^{p}}$ converges to $a_{0}+\frac{2 n q}{q-n}$. By Lemmas 3.2 and 3.6 we have

$$
\begin{aligned}
\|\chi\|_{\infty} & \leq C(q, n, A)\|X\|_{\infty}^{\frac{\gamma}{1+\gamma}}\|\chi\|_{2}^{\frac{1}{1+\gamma}} \\
& \leq C(q, n, A)\|X\|_{\infty}^{\frac{\gamma}{1+\gamma}}\left(\frac{\varepsilon}{\beta^{2}}\|X\|_{2}^{2}\right)^{\frac{1}{2(1+\gamma)}} \\
& =\frac{C_{4}(q, n, \beta, A)}{h} \varepsilon^{\frac{1}{2(1+\gamma)}} .
\end{aligned}
$$

where $\gamma=\frac{q n}{q-n}$.

Proof of Theorem 1.2. Let $\varepsilon_{0}=\varepsilon_{0}(q, n, \beta, A)>0$ such that

$$
\max \left\{C_{3} \varepsilon_{0}^{\frac{1}{2(2 n+1)}}, C_{4}^{2} \varepsilon_{0}^{\frac{1}{1+\gamma}}\right\} \leq \frac{1}{16}, \quad \text { and } \quad \varepsilon_{0} \leq \min \left(1, \beta^{2}\right)
$$

where $C_{3}=C_{3}(n, \beta, A)$ and $C_{4}=C_{4}(q, n, \beta, A)$ are the positive constants in Lemmas 3.9 and 4.2. If $\left(\Lambda_{\varepsilon}\right)$ holds with $\varepsilon \leq \varepsilon_{0}$, then we have

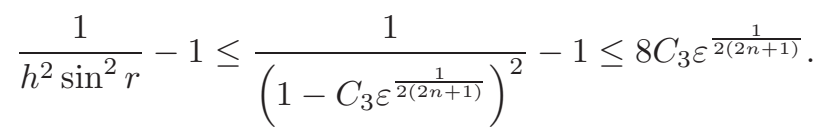

On the other hand, by Lemmas 3.9 and 4.2, we get

$$
\|\nabla r\|_{\infty}^{2} \leq \frac{h^{2}\left\|X^{\top}\right\|_{\infty}^{2}}{\left(1-C_{3} \varepsilon^{\frac{1}{2(2 n+1)}}\right)^{2}} \leq 4 C_{4}^{2} \varepsilon^{\frac{1}{1+\gamma}}
$$

which gives

$$
1-\frac{1-\|\nabla r\|_{\infty}^{2}}{h^{2} \sin ^{2} r} \leq 1-\frac{1-4 C_{4} \varepsilon^{\frac{1}{1+\gamma}}}{\left(1+C_{3} \varepsilon^{\frac{1}{2(2 n+1)}}\right)^{2}} \leq 3 C_{3} \varepsilon^{\frac{1}{2(2 n+1)}}+4 C_{4}^{2} \varepsilon^{\frac{1}{1+\gamma}} .
$$

A direct computation shows

$$
\left.|| d F_{x}(u)\right|^{2}-1 \mid \leq 8 \max \left\{C_{3} \varepsilon^{\frac{1}{2(2 n+1)}}, C_{4}^{2} \varepsilon^{\frac{1}{1+\gamma}}\right\} \leq \frac{1}{2} .
$$

Hence $F$ is a local diffeomorphism. Since $S\left(p_{0}, R_{0}\right)$ is simply connected for $n \geq 2$, then $F$ is a diffeomorphism. Therefore, $Y$ is a bijection and $\phi$ is an embedding. By Lemmas 3.9 and 4.2 , we obtain

$$
\min _{M}|X| \geq \frac{1-C_{3} \varepsilon^{\frac{1}{2(2 n+1)}}}{h}>\frac{15}{16 h}, \quad \text { and } \quad \max _{M}\left|X^{\top}\right| \leq \frac{C_{4} \varepsilon^{\frac{1}{2(1+\gamma)}}}{h}<\frac{1}{4 h},
$$

which implies that $\min _{M}\left|X^{\perp}\right|>0$. By the connectedness of $M,\langle X, \nu\rangle>0$ on $M$, which is equivalent to $\phi(M)$ is starshaped with respect to $p_{0}$. It remains to prove (1.3). A straightforward calculation gives

$$
\begin{aligned}
& \left|d_{2}\left(F\left(x_{1}\right), F\left(x_{2}\right)\right)-d_{1}\left(x_{1}, x_{2}\right)\right| \\
\leq & \left.\max _{x \in M, u \in U_{x} M}|| d F_{x}(u)\right|^{2}-1 \mid d_{1}\left(x_{1}, x_{2}\right) \\
\leq & \sqrt{2} \max \left\{C_{3}(n, \beta, A) \varepsilon^{\frac{1}{2(2 n+1)}}, C_{4}(q, n, \beta, A)^{2} \varepsilon^{\frac{1}{1+\gamma}}\right\} \operatorname{Diam}\left(S\left(p_{0}, R_{0}\right)\right) \\
\leq & \frac{\sqrt{2} \pi}{h} \max \left\{C_{3}(n, \beta, A) \varepsilon^{\frac{1}{2(2 n+1)}}, C_{4}(q, n, \beta, A)^{2} \varepsilon^{\frac{1}{1+\gamma}}\right\} .
\end{aligned}
$$

This completes the proof of Theorem 1.2. 


\section{The Optimality of Radius Condition}

In this section, we construct an example to show that the radius condition in Theorem 1.2 is optimal. In [12], Muto, Ohnita and Urakawa proved that for the great sphere $\mathbb{S}^{n}$ and the generalized Clifford torus $\mathbb{S}^{p}\left(\sqrt{\frac{p}{n}}\right) \times \mathbb{S}^{q}\left(\sqrt{\frac{q}{n}}\right)(p+q=n)$ of $\mathbb{S}^{n+1}$, the first eigenvalue $\lambda_{1}$ is just $n$. We take $M$ be one of the generalized Clifford tori, and claim that every point of $\mathbb{S}^{n+1}$ can be chosen as a center of gravity of $M$. Since the radius of $M$ is exactly $\frac{\pi}{2}$, we may choose $p_{0} \in \mathbb{S}^{n+1}$ as a center of gravity of $M$ such that $\phi(M) \subset \bar{B}\left(p_{0}, \frac{\pi}{2}\right)$. However, $M$ is not homeomorphic to $\mathbb{S}^{n}$. Now we prove the claim. It is equivalent to show that $\mathcal{E}$ is constant on $\mathbb{S}^{n+1}$. We fix $p \in\left\{1, \cdots,\left[\frac{n}{2}\right]\right\}$ and set $q=n-p$.

$$
M=\mathbb{S}^{p}\left(r_{1}\right) \times \mathbb{S}^{q}\left(r_{2}\right) \stackrel{\phi}{\hookrightarrow} \mathbb{S}^{n+1} \stackrel{i}{\hookrightarrow} \mathbb{R}^{n+2},
$$

where $r_{1}=\sqrt{\frac{p}{n}}$ and $r_{2}=\sqrt{\frac{q}{n}}$.

By the spherical coordinates $\left(\theta_{1}, \cdots, \theta_{p}, \beta_{1}, \cdots, \beta_{q}\right)$, we have

$$
\left\{\begin{array}{c}
x_{1}=r_{1} \sin \theta_{1} \cos \theta_{2} \cdots \cos \theta_{p-1} \cos \theta_{p}, \\
x_{2}=r_{1} \sin \theta_{1} \cos \theta_{2} \cdots \cos \theta_{p-1} \sin \theta_{p}, \\
\vdots \\
x_{p}=r_{1} \sin \theta_{1} \sin \theta_{2}, \\
x_{p+1}=r_{1} \cos \theta_{1}, \\
x_{p+2}=r_{2} \sin \beta_{1} \cos \beta_{2} \cdots \cos \beta_{q-1} \cos \beta_{q}, \\
x_{p+3}=r_{2} \sin \beta_{1} \cos \beta_{2} \cdots \cos \beta_{q-1} \sin \beta_{q}, \\
\vdots \\
x_{p+q+1}=r_{2} \sin \beta_{1} \sin \beta_{2}, \\
x_{p+q+2}=r_{2} \cos \beta_{1},
\end{array}\right.
$$

where $\theta_{1}, \beta_{1} \in[0, \pi)$ and $\theta_{2}, \cdots, \theta_{p}, \beta_{2}, \cdots, \beta_{q} \in[0,2 \pi)$. A direct computation gives

$$
\sqrt{\operatorname{det} g}=r_{1}^{p} r_{2}^{q}\left(\sin \theta_{1}\right)^{p-1}\left(\sin \beta_{1}\right)^{q-1} \prod_{j=2}^{p-1}\left(\cos \theta_{j}\right)^{p-j} \prod_{k=2}^{q-1}\left(\cos \beta_{k}\right)^{q-k} .
$$

For any point $p=\left(p_{1}, \cdots, p_{n+2}\right) \in \mathbb{S}^{n+1} \hookrightarrow \mathbb{R}^{n+2}$, we have

$$
\begin{aligned}
\mathcal{E}(p) & =\int_{M}[1-\cos d(p, x)] d v(x)=\int_{M}\left(1-\sum_{i=1}^{n+2} p_{i} x_{i}\right) d v(x) \\
& =\int_{[0, \pi)^{2} \times[0,2 \pi)^{n-2}}\left(1-\sum_{i=1}^{n+2} p_{i} x_{i}\right) \sqrt{\operatorname{det} g} d \theta_{1} d \beta_{1} d \theta_{2} \cdots d \theta_{p} d \beta_{2} \cdots d \beta_{q} \\
& =|M| .
\end{aligned}
$$

Therefore, $\mathcal{E}$ is constant on $\mathbb{S}^{n+1}$.

\section{REFERENCES}

[1] Aubry E., Grosjean J.F., and Roth J., Hypersurfaces with small extrinsic radius or large $\lambda_{1}$ in Euclidean spaces, arXiv:1009.2010v1. 
[2] Brendle S., Minimal surfaces in $\mathbb{S}^{3}$ : a survey of recent results, Bull. Math. Sci. 3(2013), 133-171.

[3] Chavel I., Riemannian geometry-A modern introduction, Cambridge Studies in Advanced Math., Vol.102, Cambridge Univ. Press, New York, 2006.

[4] Choi H.I. And Wang A.N., A first eigenvalue estimate for minimal hypersurfaces, J. Differential Geom. 18(1983), 599-602.

[5] Colbois B. and Grosjean J.-F., A pinching theorem for the first eigenvalue of the Laplacian on hypersurfaces of the Euclidean space, Comm. Math. Helv. 82(2007), 175-195.

[6] Grosjean J.F. Upper bounds for the first eigenvalue of the Laplacian on compact submanifolds, Pacific J. Math. 206(2002), 93-112.

[7] Grosjean J.F. and Roth J. Eigenvalue pinching and application to the stability and the almost umbilicity of hypersurfaces, Math. Z. 271(2012), 469-488.

[8] Heintze E., Extrinsic upper bound for $\lambda_{1}$, Math. Ann. 280(1988), 389-402.

[9] Hoffman D. and Spruck J., Sobolev and isoperimetric inequalities for Riemannian submanifolds, Comm. Pure Appl. Math. 27(1974), 715-727. Erratum, Comm. Pure Appl. Math. 28(1975), 765-766.

[10] Hu Y.X., Xu H.W. ANd Zhao E.T., First eigenvalue pinching for Euclidean hypersurfaces via $k$-th mean curvatures, Ann. Glob. Anal. Geom. 48(2015), 23-35.

[11] Jorge L. and Xavier F., An inequality between the exterior diameter and the mean curvature of bounded immersions, Math. Z. 178(1981), 77-82.

[12] Muto H., Ohnita Y. and Urakawa, Homogeneous minimal hypersurfaces in the unit spheres and the first eigenvalues of their Laplacian, Tohoku Math. J. 36(1984), 253-267.

[13] Myers S., Curvature of closed hypersurfaces and non-existence of closed minimal hypersurfaces, Trans. Amer. Math. Soc. 71(1951), 211-217.

[14] Petersen P., Riemannian Geometry (second edition), Graduate Texts in Mathematics, Vol.171, Springer, New York, 2006.

[15] Reilly R., On the first eigenvalue of the Laplacian for compact submanifolds of Euclidean space, Comm. Math. Helv. 52(1977), 525-533.

[16] Rотн J., Extrinsic radius pinching for hypersurfaces of space forms, Differential Geom. and its Appl. 25(2007), 485-499.

[17] Rотн J., Extrinsic radius pinching in space forms of nonnegative sectional curvature, Math. Z. 258(2008), 227-240.

[18] Roth J., Pinching of the first eigenvalue of the Laplacian and almost-Einstein hypersurfaces of the Euclidean space, Ann. Glob. Anal. Geom. 33(2008), 293-306.

[19] Schoen R. and Yau S.T., Lectures on Differential Geometry, Conference Proceedings and Lecture Notes in Geometry and Topology I, International Press, Cambridge, 1994.

[20] El Soufi A. And Ilias S., Une inegalité du type "Reilly" pour les sous-variétés de l'espace hyperbolique, Comm. Math. Helv. 67(1992), 167-181.

[21] Veeravalli A., On the first Laplacian eigenvalue and the center of gravity of compact hypersurfaces, Comm. Math. Helv. 76(2001), 155-160.

[22] Takahashi T., Minimal immersions of Riemannian manifolds. J. Math. Soc. Japan 18(1966), 380-385.

[23] TANG Z.Z. AND YAN W.J., Isoparametric foliation and Yau conjecture on the first eigenvalue, J. Differential Geom. 94(2013), 521-540.

[24] Tang Z.Z., Xie Y.Q. and Yan W.J., Isoparametric foliation and Yau conjecture on the first eigenvalue, II, J. Funct. Anal., 266(2014), 6174-6199.

[25] YAu S.T., Problem section, In: Seminar on Differential Geometry, Ann. of Math. Studies, Vol. 102, Princeton University Press, Princeton, 1982, 669-706.

Center of Mathematical Sciences, Zhejiang University, Hangzhou 310027, China,

E-mail address: huyx@cms.zju.edu.cn, xuhw@cms.zju.edu.cn 